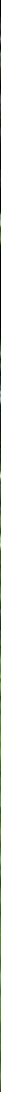

\title{
Espacios públicos amables para las mujeres en Xalapa, Veracruz, una mirada desde el Observatorio Urbano Universitario
}

\author{
H. Rubio ${ }^{1}$ - M. Hernández ${ }^{2}$ \\ C. Martínez ${ }^{3}$ - M. M. Ferrer ${ }^{4}$
}

RESUMEN: Desde la Arquitectura y el Urbanismo, poco se ha abordado la perspectiva de género en el diseño y estudio del espacio urbano. En su gran mayoría, las ciudades han sido diseñadas desde una perspectiva neutral, en donde poco se han considerado las necesidades de las mujeres. Se aborda, el estudio del espacio público considerando una mirada femenina a la problemática que vive este grupo en el uso, apropiación y percepción del espacio público, frente a las condiciones de inseguridad que se viven en las ciudades. En este caso se analiza la ciudad de Xalapa, a través de una metodología mixta, este trabajo tiene como meta proveer de datos, así como recomendaciones que sirvan de fundamento para la toma de decisiones en la creación de ciudades más seguras a través de un urbanismo con perspectiva de género.

Palabras Clave: ciudades, violencia, perspectiva de género, espacio público, Xalapa-México.

ABSTRACT: In Architecture and the Urbanism, the study and design of the urban space has little been approached from a gender perspective. Urban space and its public spaces have been designed in their great majority from a neutral perspective, where the needs of the women have hardly been considered. In this article, the study of public space is approached, considering a feminine point of view of the problems this social group experience in the use, appropriation and perception of publics spaces, under the insecurity conditions of cities. In this case, Xalapa one of the most insecure cities in the state of Veracruz-Mexico is analyzed. Through a mixed methodology, this work aims to provide data, as well as recommendations that serve as a basis for decision making in the creation of safer cities through urban planning with a gender perspective.

Keywords: cities, violence, gender perspective, public space, Xalapa-México. 


\section{Introducción}

$\square$ 1 recrudecimiento de la inseguridad ha tomado por sorpresa a la población veracruzana. Sus esquirlas han diezmado la dinámica y desarrollo económico, el derecho al libre tránsito por el espacio público, así como la vida de un número aún no determinado de habitantes del estado (Casados 2017). En este contexto, se declaró en noviembre de 2016 la "Alerta por Violencia de Género" para 11 municipios del Estado de Veracruz, entre ellos Xalapa; a partir de esto se hace necesario implementar acciones de recuperación de espacios públicos que garanticen la seguridad, libertad y bienestar de las mujeres en la ciudad; cualidades que deben generarse con el diseño y configuración de los espacios en conjunto con acciones y políticas ciudadanas.

Es importante mencionar que la "Ley de Igualdad entre Hombres y Mujeres de la Universidad Veracruzana", señala que las Facultades, Institutos y Centros de investigación deben contribuir con la comunidad a garantizar la igualdad, la no violencia y no discriminación en sus entornos; y deben integrar la perspectiva de género en las funciones sustantivas de la universidad: docencia, investigación, gestión. Por esto, la reflexión acerca de una nueva forma de observar la arquitectura y la ciudad, aplicando la perspectiva de género en la configuración espacial, así como el entendimiento, diseño y transformación de los entornos urbanos y rurales del Estado de Veracruz, es una labor que, actualmente se está fortaleciendo en la Universidad Veracruzana.

Es por esto que surge el proyecto "Espacios Públicos Amables para las Mujeres de Xalapa, Veracruz", que es un proyecto de investigación, docencia y gestión de la Universidad Veracruzana, en el que se vinculan la Facultad de Arquitectura, los cuerpos académicos "Entornos Sustentables" y "Género y Cultura", El Obser- vatorio Urbano Universitario, La CoSustenta y la esencial participación del Instituto Municipal de las Mujeres de Xalapa, de quien surgió la solicitud expresa de atender el tema en los espacios públicos para contribuir a las diversas respuestas y soluciones de la Alerta por Violencia de Género en Xalapa. El objetivo principal del proyecto es investigar y analizar las condiciones de habitabilidad, la configuración y ubicación de los lugares y los fenómenos que hacen a los espacios públicos de Xalapa lugares agresivos, violentos y discriminatorios para las mujeres; pero también aquellas características de los espacios públicos que los hacen entrañables, seguros y positivamente significativos para ellas; a fin de generar criterios de diseño espacial, que contribuyan a crear espacios públicos amables y seguros para todas y todos. Este artículo presenta la primera etapa del proyecto, que es un diagnóstico de la ciudad de Xalapa, a partir de una metodología combinada entre investigación cartográfica, demográfica y observación del entorno urbano. En donde se analizan a escala ciudad cómo se manifiesta la violencia de género, así como la inseguridad en los diversos sectores y colonias y el espacio público Xalapeño.

\section{Ciudad, violencia y género}

La violencia contra las mujeres y niñas en las ciudades veracruzanas, ha ido creciendo en número y en complejidad, haciendo que se vuelva urgente atender esta problemática desde de varios ámbitos institucionales, ciudadanos y universitarios con una perspectiva inter, multi y transdisciplinaria. Además, se suman los conflictos urbanos que de manera general afectan a la población de Xalapa, como lo son; la alta concentración de vehículos, el poco espacio y respeto para las formas alternativas de movilidad más sustentables, el creciente daño

1 Arquitecta, Doctora en Estudios Urbanos por la Universidad Autónoma Metropolitana (México), integrante del Observatorio Urbano Universitario y del CA-Entornos Sustentables. Correo: harubio@uv.mx

2 Arquitecto, Doctor en Planificación y Paisaje Urbano por la Universidad de Newcastle upon Tyne (Inglaterra) coordinador del Observatorio Urbano Universitario y del CA- Entornos Sustentables. Correo: maurhernandez@uv.mx

3 Asistente de investigación, Estudiante de la Facultad de Arquitectura-Xalapa, Universidad Veracruzana.

4 Asistente de investigación, Estudiante de la Facultad de Arquitectura-Xalapa, Universidad Veracruzana.

UVserva No. 5 Noviembre 2017 - Abril 2018 
en el entorno natural, y la poca valoración que se les da a los espacios públicos como patrimonio comunitario. Aunque a simple vista estas problemáticas urbanas y la de violencia contra las mujeres no tienen puntos de contacto, sí existen nexos importantes; ya que ellas al igual que los otros grupos (hombres, niños y niñas, personas mayores) representan actores importantes en el uso cotidiano de la ciudad.

Consideramos que al abordar el urbanismo con perspectiva de género, en el diagnóstico, los proyectos, y finalmente las políticas públicas en los temas de espacio público, se está contribuyendo a solucionar el problema desde la raíz, ya que se atiende a la diversidad de maneras de experimentar, percibir y conocer los espacios de la ciudad desde una mirada femenina; la cual hasta hoy ha sido ignorada. De aquí, que el proyecto tiene un enfoque sustentable y feminista, del cual surge una postura crítica que cuestiona la neutralidad de los espacios de la ciudad y se plantea que más bien son percibidos, experimentados y entendidos de acuerdo al género y a la etapa de vida de las personas; sin embargo, se han diseñado bajo la mirada de una élite patriarcal (Durán 2000, Bolos 2008, Falú 2009, Muxi 2015).

A partir de la vida en el espacio público: de la presencia, experiencia y percepción de las mujeres en él; podemos comprender en gran medida el nivel de violencia que se ejerce contra ellas. En noviembre del año 2016 se llega a un momento crítico con respecto a la violencia contra las mujeres y se declara en el estado de Veracruz la "Alerta de violencia de género" en doce municipios, entre ellos Xalapa. Se incrementa la cantidad de feminicidios, maltrato y violencia de todos tipos. No obstante, estos he- chos se presentan también en el ámbito rural, es en las ciudades y en los grandes conglomerados urbanos donde se hace más evidente.

Una de las manifestaciones más graves de esta desigualdad en la ciudad es la violencia que sufren las mujeres cotidianamente en el espacio público, donde son acosadas, agredidas y discriminadas continuamente. Esta violencia refleja que todavía se les sigue negando el carácter de ciudadanas, precisamente en uno de los lugares más importantes donde debe materializarse: La calle, el espacio público primigenio y más emblemático de la ciudad. No obstante, a partir de la segunda mitad del siglo XX en México y otros países, las mujeres han salido más a la calle y la han ido convirtiendo en parte de su cotidianeidad, como lugar de circulación, de trabajo, de descanso o de encuentro. Pero aún no se dan totalmente las condiciones para que las mujeres disfruten de igual manera de los espacios públicos como los hombres. Las ciudades mexicanas están creadas y diseñadas en la lógica del sistema hetero-patriarcal; de tal manera que las mujeres se han de adaptar a la forma, funciones y dinámicas que esta ciudad ajena a ellas, les ofrece.

En el Estado de Veracruz según el periodo intercensal del 2015 del INEGI, las mujeres son el 50\% de la población, las cuales en su gran mayoría pagan impuestos desde el momento que trabajan o compran algún artículo. El espacio público se construye en su parte material con estos recursos, sin embargo, cuando se toman decisiones acerca de la creación, transformación e intervención de los espacios públicos, rara vez se toma en cuenta la realidad y la perspectiva de las mujeres. Hombres y mujeres en general, no percibimos, y tam-

Tabla 1. Casos de violencia contra las mujeres primer semestre del 2017

\begin{tabular}{|c|c|c|c|c|c|c|c|}
\hline & Enero & Febrero & Marzo & Abril & Mayo & Junio & Totales \\
\hline Región Norte & 12 & 12 & 8 & 16 & 8 & 10 & 66 \\
\hline Región Centro & 16 & 9 & 14 & 10 & 9 & 11 & 69 \\
\hline Región Sur & 57 & 54 & 65 & 69 & 77 & 42 & 364 \\
\hline Estatal & 85 & 75 & 87 & 95 & 94 & 63 & 499 \\
\hline
\end{tabular}

Fuente: Observatorio de violencias contra las mujeres. 
poco experimentamos la ciudad de la misma manera. Como ya lo hemos dicho, los lugares de la ciudad no son neutros, están diseñados para un modelo de ciudadano con características específicas, que son por lo general, burgués, blanco, varón y adulto. Todas las personas que no cumplen la mayor parte de estas características (mujeres, niñas y niños, ancianos, personas con alguna discapacidad, diversas etnias indígenas, etc.), tienen que adaptarse a formas del espacio, usos y elementos urbanos que no están diseñados de acuerdo a sus necesidades.

Por otro lado, dependiendo de los roles de género que se nos han asignado a hombres y mujeres, de los mitos y ritos que se han heredado en la manera de utilizar los espacios, la violencia que se ejerce hacia las mujeres se manifiesta en un uso y una percepción diferenciada de los lugares de la ciudad. Todas estas ideas ya han sido abordadas por investigadoras como María Ángeles Durán o Zaida Muxi. La problemática que aquí se aborda es de origen político. Dice Silvia Bolos (2008) que en la "no reproducción", es decir en la sexualidad, es donde se anuda el tema de la ciudadanía de las mujeres; y el tema de la ciudadanía es central cuando se pretende entender el espacio público. Las mujeres no han sido reconocidas como ciudadanas ni como sujetas de derecho y de opinión. Es decir, se ven únicamente como reproductoras, como responsables de la crianza y el cuidado de todos los seres de la comunidad, pero no como tomadoras de decisiones ni como artistas, pensadoras o productoras de ideas. Así pues, cuando una mujer está en la calle y no está ejerciendo el mandato y rol de cuidadora o reproductora, es una mujer a la que se le vuelve aún más difícil su estancia, paso y participación en las calles de la ciudad.

\section{Metodología de la investigación}

La metodología utilizada involucra la perspectiva de género al estudio y diagnóstico de los espacios públicos de la ciudad. Dicha perspectiva es pertinente considerar con el afán de introducir otros enfoques a la arquitectura y el urbanismo que no habían sido considerados en el diseño y configuración de espacios urbanos. Esta metodología aplica tres conceptos básicos de la vida en el espacio público que hay que valorar para garantizar que una ciudad sea amable con las mujeres, y que hemos retomado de las aportaciones teóricas y metodológicas de María Ángeles Durán, Zaida Muxí y el Colectivo de urbanismo feminista Punt 6 de Barcelona. Los tres conceptos a considerar son: uso, percepción y apropiación pero teniendo como actor clave las mujeres. Con relación al uso, el principal cuestionamiento es ¿Que hacemos en los espacios y cómo los usamos? En cuanto a percepción, nos cuestionamos ¿Cómo percibimos el espacio. Respecto al tema de la apropiación, la pregunta es ¿De qué manera nos vinculamos con el espacio y lo hacemos propio?. Bajo estos tres ejes fundamentales, se organizó la información de los espacios públicos de la ciudad de Xalapa, a través del estudio urbano mediante Sistemas de Información geográfica (SIG) se llevó a cabo una investigación de corte socio-espacial de acuerdo a datos de distintas Instituciones y organismos, y también se realizaron encuestas y análisis de las redes sociales Facebook e Instagram de corte cualitativo para conocer opiniones y percepciones sobre las tres temáticas fundamentales. En este artículo describiremos la primera etapa del proyecto, y reflexionaremos los datos encontrados en un primer acercamiento a la violencia contra las mujeres en el territorio de la ciudad de Xalapa.

Diagnóstico violencia, inseguridad urbana y mujeres: el caso Xalapa, Ver.

Para poder dar inicio a un proyecto urbano en el cual se pueda proponer un espacio público amable para las mujeres, antes que nada debemos analizar la ciudad de Xalapa, ver dónde y cómo la utilizan las mujeres. Uno de los principales argumentos que planteamos en este trabajo de investigación es que los actos de violencia hacia las mujeres se generan en los trayectos que recorren del trabajo a sus casas o viceversa, por lo cual se hace necesario un análisis de la población femenina de la ciudad de Xalapa. 
Figura 1. Análisis demográfico y Centros Económicos de Xalapa

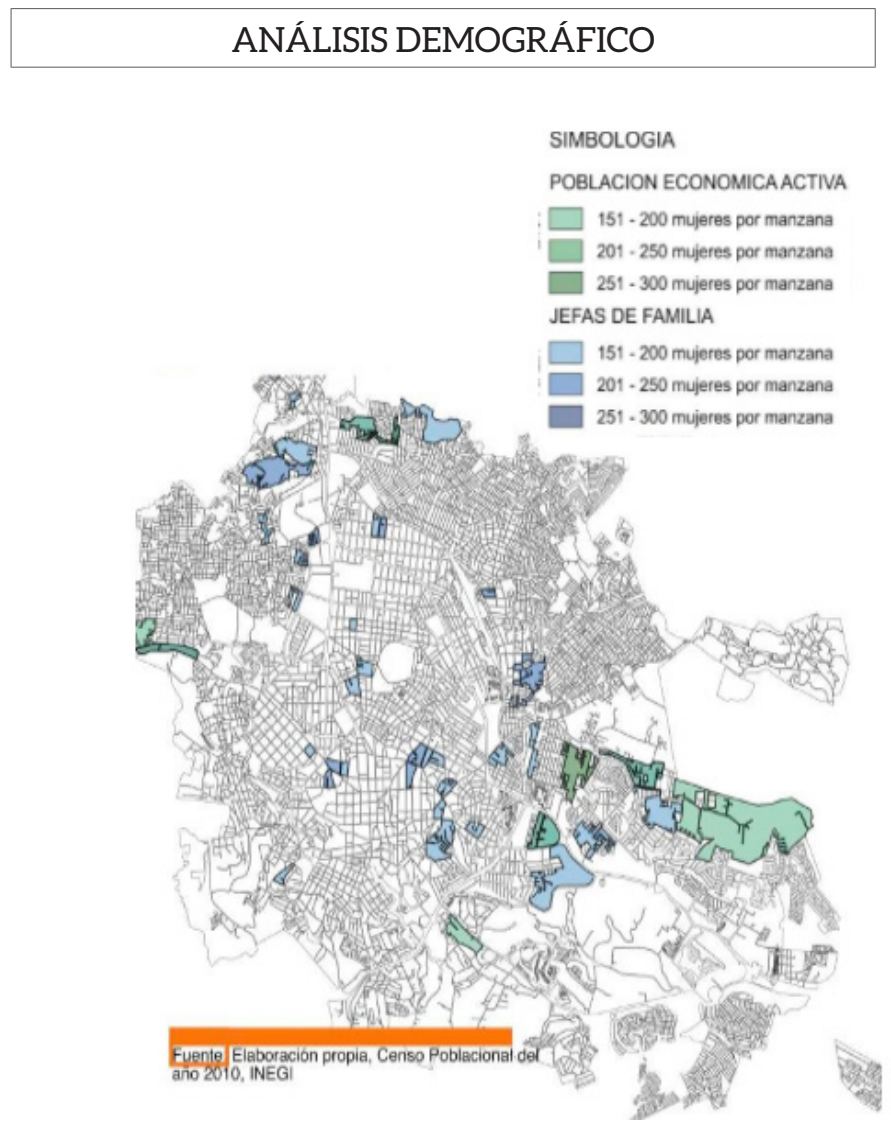

Fuente: Elaboración propia, Censo Poblacional del año 2010, INEGI.

En el censo poblacional 2010, realizado por el INEGI, se tiene como resultado que Xalapa tiene una población total de 457, 928 habitantes de los cuales el 47\% (213, 571 habitantes), son hombres y un 53\% (244, 357 habitantes), son mujeres. A partir de aquí podemos analizar dos indicadores del censo: la Población Femenina Económicamente Activa y las Jefas de Familia, que corresponden a un total de 84,090 habitantes y 43,172 habitantes, respectivamente. Hay que mencionar que una misma mujer puede entrar en ambos indicadores. En la figura 1 podemos visualizar cómo está distribuida la población femenina en el territorio de la ciudad de Xalapa con relación a los rangos de habitantes por manzana más altos, que van de 151 a 200, de 201 a 250 y de 251 a 300, los cuales además muestran los lugares en dónde viven las mujeres de ambos indicadores.

Por otro lado, consideramos que es importante conocer a qué lugares se trasladan para trabajar o para realizar cualquier actividad. En
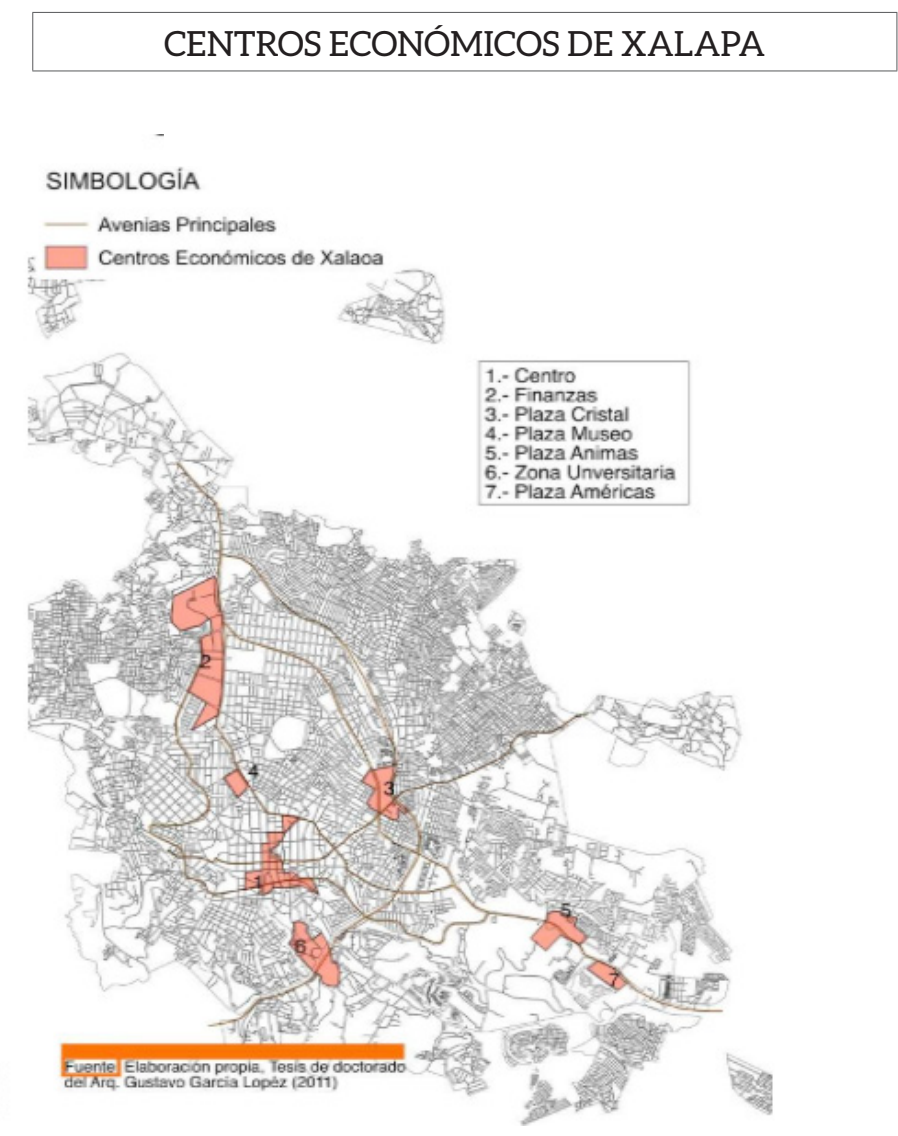

Fuente: Elaboración propia, Tesis de doctorado del Arq. Gustavo García López (2011).

este sentido los principales centros económicos de Xalapa (figura 2 ) juegan un papel importante, ya que ahí se concentran una buena parte de la economía femenina de la ciudad y en algunos lugares coinciden con sitios de entrada y salida de la población que viene de otros lados de la ciudad, como Plaza Cristal (lugar a donde llegan personas del Castillo, Alto Lucero, Actopan, etc.); en la zona de la Secretaría de Finanzas (en donde se concentran paradas de autobuses que vienen de Banderilla, Perote, Naolinco, Misantla, etc.); estos son dos nodos de entrada y salida de la ciudad de Xalapa. Existen otros puntos, que por las diversas actividades urbanas que ahí se desarrollan resultan ser nodos de concentración poblacional importantes como lo son el centro de la ciudad, la zona universitaria, la zona de plaza Américas y Animas, la zona del Mercado los Sauces así como la zona del mercado de la Rotonda. En general son puntos en donde la población femenina se mueve en dirección al trabajo o vivienda. 


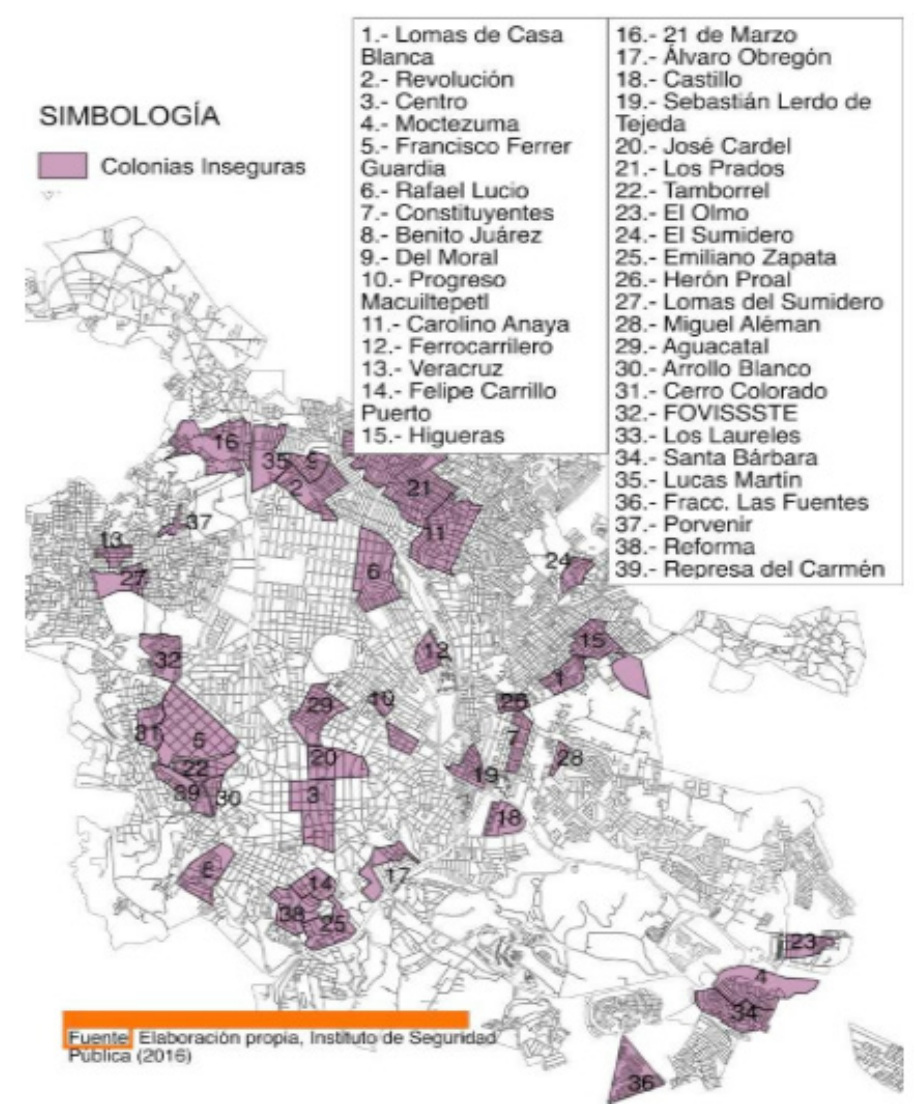

Fuente: Elaboración propia, Instituto de Seguridad Pública (2016).

En este contexto, también se identificaron la situación de la inseguridad en las colonias y a através de la información obtenida de instituciones como el Instituto Municipal de Seguridad Pública, encontramos que existen 39 colonias, en las cuales se han suscitados actos delictivos contra las mujeres (ver figura 3 y 4). Por otro lado, a través de la información obtenida del Instituto Municipal de las Mujeres de Xalapa, podemos identificar en la ciudad los casos de violencia atendidos por este Instituto y territorializar esta información, en cuanto a su distribución en el contexto urbano Xalapeño. De ahí que en 2016 podemos identificar que tanto en las delegaciones Norte y Centro-Sur de la capital del estado es donde se localizaron la mayoría de los casos atendidos por diversos tipos de violencia. En la delegación norte se presentaron 135 casos y en la delegación Centro-Sur 170 casos. En la primera se proporcionó atención en cuanto a Prevención y Atención a la Violencia (118 casos) y Atención Psicológica y Salud Integral (17 casos) y en la segunda se proporcionó atención en cuanto a Prevención y Atención a la Violencia (136 casos) y Atención Psicológica y Salud Integral (34 casos). De esta manera se identifican las colonias que presentan un alto índice de inseguridad y de violencia contra las mujeres: la Colonia Veracruz, colonia Plan de Ayala y la colonial el Moral, las tres localizadas al Norte de la ciudad (Tabla 2).

Para conocer más sobre la situación de violencia e inseguridad que se vive en la ciudad de Xalapa también se obtuvo información del Observatorio Universitario de Violencias contra las Mujeres, sobre los casos de feminicidios registrados por este observatorio en donde se identifican las colonias (figura) que presentaron al menos un evento de feminicidio durante 2016. De aquí podemos cruzar la información de las diversas fuentes para concluir que se identifican coincidencias en algunas colonias relacionadas a actos delictivos contra las mujeres y eventos de feminicidio. Los datos son 
Figura 3. Colonias con al menos un caso de feminicidio en 2016 y espacios públicos inseguros COLONIAS CON AL MENOS UN CASO DE FEMINICIDIO EN 2016

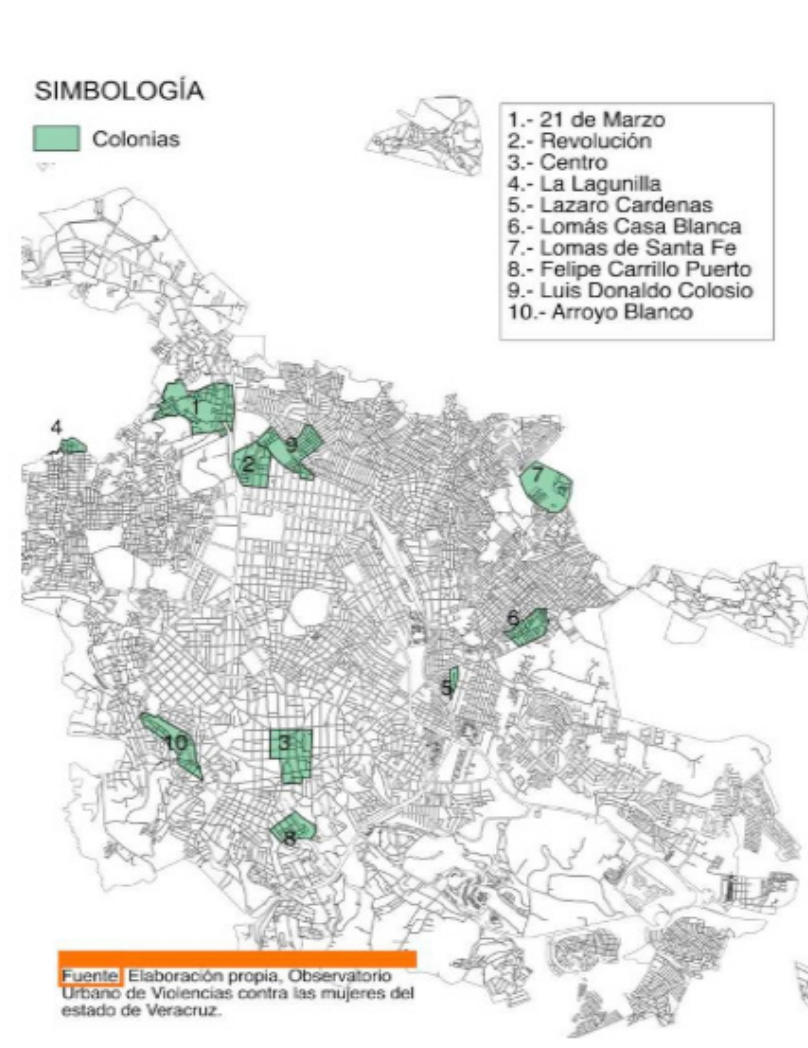

Fuente: Elaboración propia, Observatorio Universitario de Violencias contra las Mujeres del Estado de Veracruz.

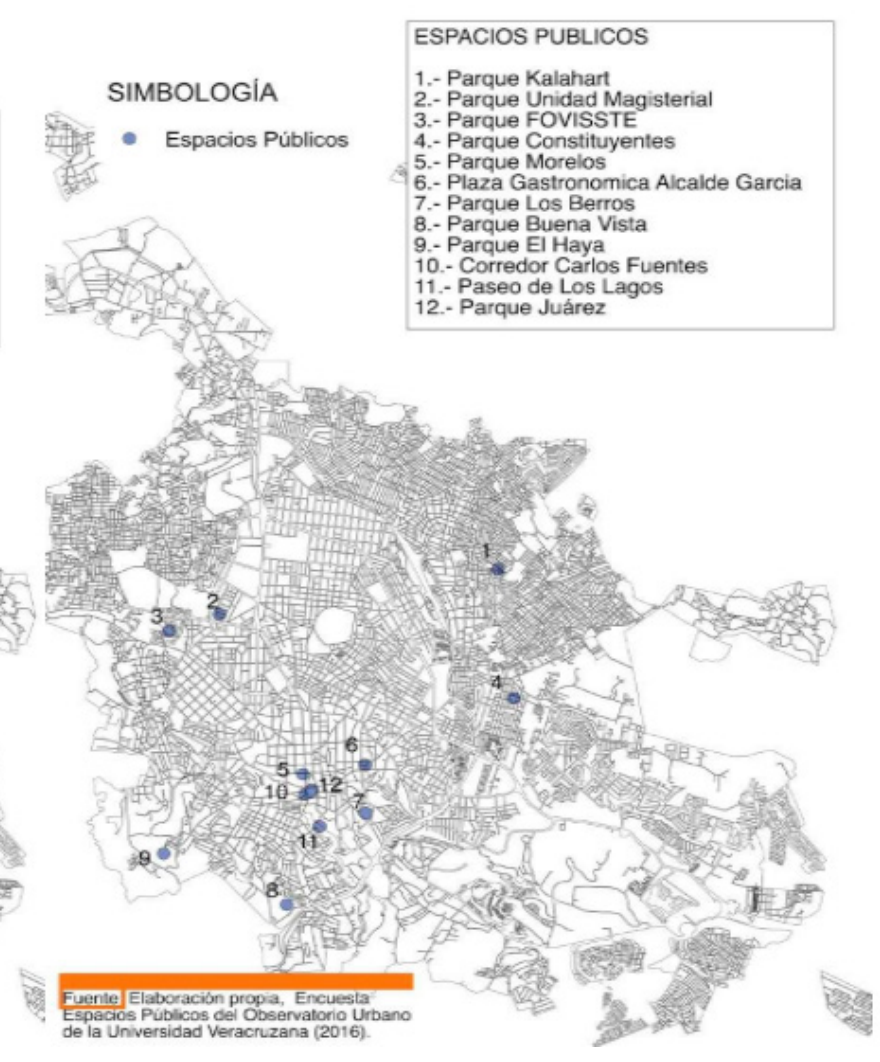

Fuente: Elaboración propia, Encuesta de espacios públicos, del Observatorio Urbano Universitario (2016).

Tabla 2. Casos de violencia atendidos por el Instituto municipal de las mujeres en 2016

\begin{tabular}{|c|c|c|}
\hline Tipo de atención & $\begin{array}{c}\text { Delegación Norte } \\
135 \text { casos }\end{array}$ & $\begin{array}{c}\text { Delegación Centro-Sur } \\
170 \text { casos }\end{array}$ \\
\hline Prevención y Atención a la Violencia & 118 & 136 \\
\hline Atención Psicológica y Salud Integral & 17 & 34 \\
\hline
\end{tabular}

Fuente: Elaboración propia.

fundamentales para identificar la localización del grado máximo de violencia contra las mujeres que se vive en Xalapa.

Para complementar la investigación espacio-territorial de la violencia contra las mujeres en Xalapa, nos basamos en trabajos anteriores desarrollados al interior del Observatorio Urbano Universitario, con relación a la investigación sobre el rescate de los espacios públicos de la ciudad de Xalapa en el periodo (2000-2016), en donde, entre otros aspectos se analiza la percepción de inseguridad que tienen los usuarios de los diversos espacios públicos de la ciudad. De aquí se identifican los motivos (ej. poca iluminación, porque casi no hay gente, por que se han presentado actos violentos, porque hay mucha basura, por un mal mantenimiento, entre otros) que las mujeres consideran cuando sienten inseguridad en los espacios públicos. Aquí, podemos identificar que diversos espa- 
Figura 4. Espacios públicos seguros e inseguros

\section{ESPACIOS PÚBLICOS SEGUROS}

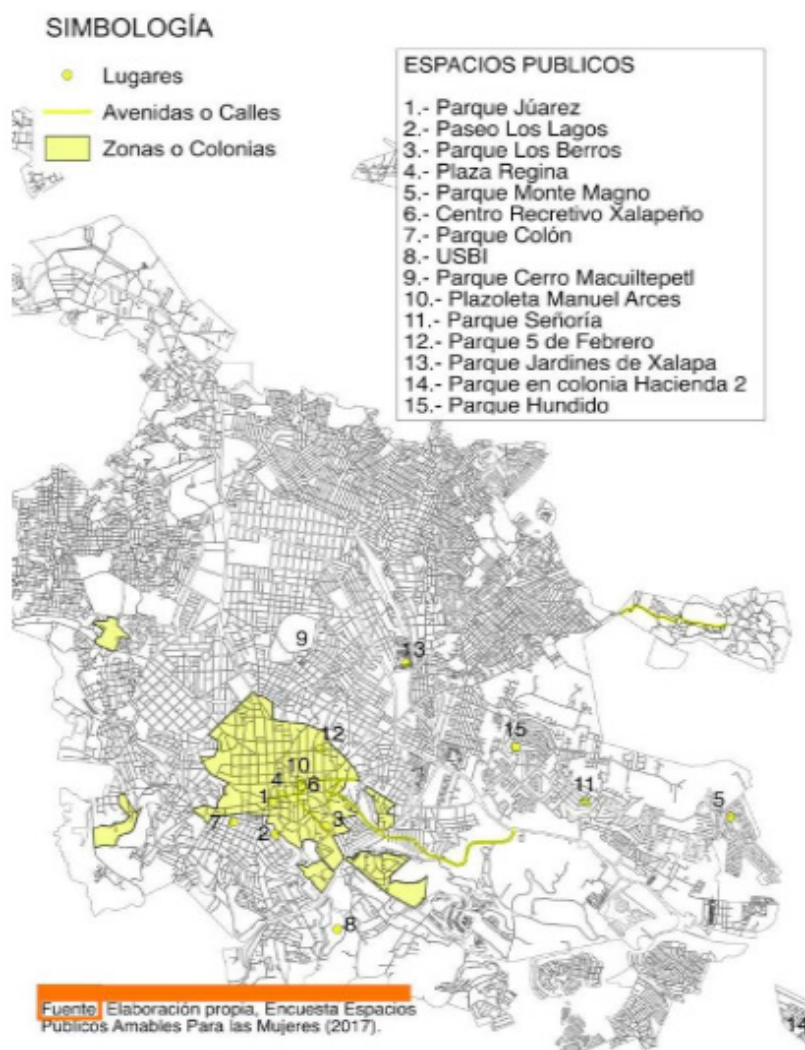

Fuente: Elaboración propia, Encuesta de espacios públicos amables para las mujeres (2017).

cios de la zona Centro-sur de la ciudad son percibidos como inseguros.

\section{La percepción de las mujeres}

Dado a que actualmente las redes sociales constituyen una manera en la cual es posible obtener datos acerca de la percepción de las personas acerca de diversos temas, se decidió hacer uso de éstas para investigar cómo las mujeres perciben los espacios públicos de la ciudad. De esta forma, se hizo uso de Facebook e Instagram, dos de las redes sociales más usadas en la actualidad; Se consultaron alrededor de 70 mujeres de diferentes edades entre los 18 y 50 años, y se abordó a través de ellas, la perspectiva metodológica de este proyecto que involucra los tres conceptos para estudiar los espacios públicos desde la perspectiva de géne-
ESPACIOS PÚBLICOS INSEGUROS

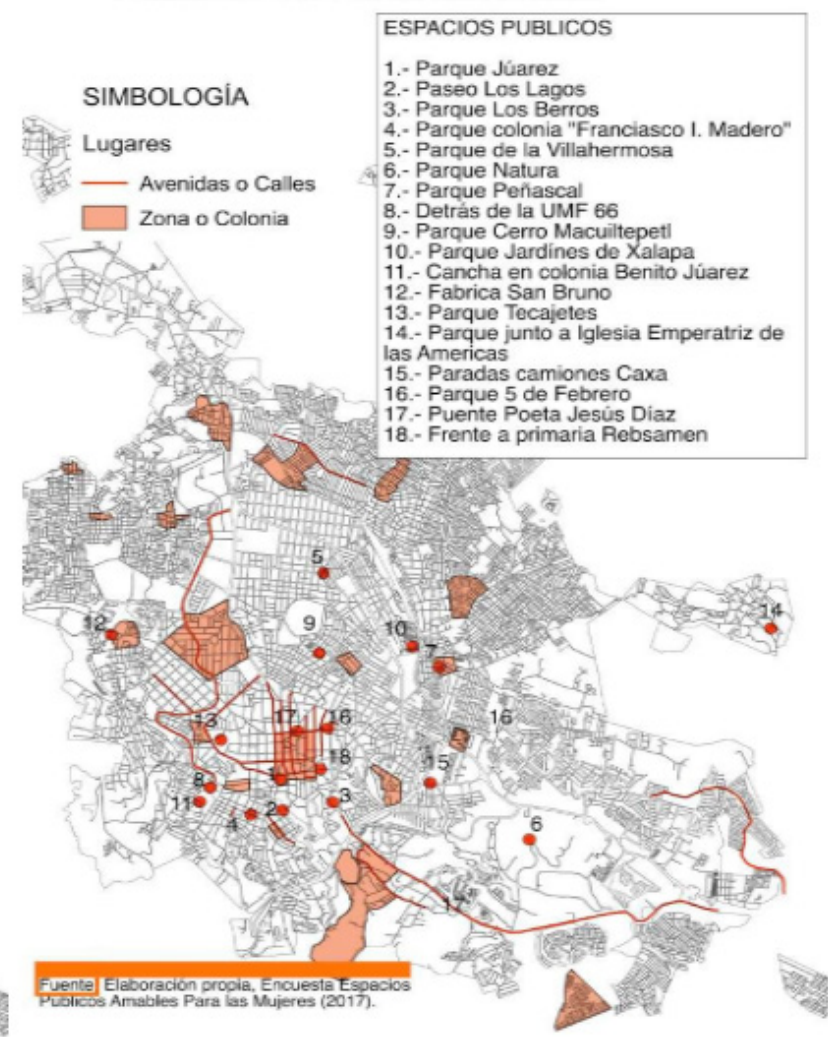

Fuente: Elaboración propia, Encuesta de espacios públicos amables para las mujeres (2017).

ro: uso, percepción y apropiación.

Si bien es cierto que las usuarias de Facebook no se encuentran en todos los sectores de la ciudad, ni son representativas de todos los rangos de edades y ocupaciones; sí pudimos obtener una radiografía que nos da un punto de partida en la percepción de las habitantes de Xalapa; que se complementará en una segunda etapa de este proyecto a partir de entrevistas a profundidad y talleres participativos en zonas estratégicas de la ciudad.

En este sentido se obtuvieron datos en cuanto a los espacios más frecuentados por las mujeres dentro de la ciudad, son calles y espacios de esparcimiento de la zona centro de la ciudad. Por ejemplo el parque Juárez, el Paseo de los Lagos y el Parque de los Berros son los espacios más mencionados. Los motivos por los que son estos los lugares más votados es porque se encuentran de paso hacia el trabajo, ha- 
cia el hogar o hacia las zonas de consumo de insumos de las mujeres encuestadas, también por ser lugares transitados por más gente de Xalapa y ser los de preferencia para el esparcimiento, exceptuando casos de parques que se encuentran en la periferia de la ciudad que son frecuentes por ser los más cercanos al lugar de residencia de las encuestadas.

En este sentido, la zona en la que convergen más espacios públicos percibidos seguros por las mujeres es el centro de la ciudad. Podría deducirse que por ser espacios públicos céntricos son los más visitados y conocidos y por esto se consideran seguros; sin embargo, también la zona centro fue considerada insegura, y algunos de los espacios considerados seguros durante el día, son considerados como inseguros durante la noche, cuando hay poca actividad, o bien porque están poco iluminados. Aquí también se mencionan espacios más periféricos como el Parque Natura, el Cerro del Macuiltepetl, entre otros ubicados en colonias consideradas como peligrosas (Figura 4).

\section{Conclusiones preliminares}

Este es un panorama general de la experiencia de las mujeres en los espacios públicos de la ciudad de Xalapa, el proyecto y la exploración de los espacios públicos con una perspectiva y género continuará con un trabajo más detallado en las colonias identificadas como más inseguras en la ciudad: la Colonia Veracruz, la Plan de Ayala y la El Moral además se abordará el proyecto desde una perspectiva inter y transdisciplinaria en conjunto con las habitantes de la ciudad. Xalapa es una ciudad que se ha construido desde la planeación oficial y desde su crecimiento informal, sin perspectiva de género. La experiencia de las mujeres, su vida cotidiana y los riesgos que ellas viven al recorrer las calles, no se toman en cuenta para decidir acerca del diseño de los espacios de la ciudad. Además, no existe aún en México la legislación específica que marque las pautas para construir calles y barrios seguros y amables desde la perspectiva de género. Los hechos y estadísticas proporcionadas por instituciones y organismos como la Secretaria de Seguridad Pública, el Observatorio Universitario de Violencia contra las Mujeres o el Instituto Municipal de las Mujeres, dan cuenta de una situación alarmante que requiere acciones multidimensionales. En este trabajo se pretende hacer una aportación desde la Arquitectura y el Urbanismo, sin tener una posición determinista entre las relaciones entorno y conducta humana y en este caso de conductas delictivas, antisociales y de violencia contra las mujeres. Es claro que el comportamiento de las personas y el ambiente son elementos que interactúan mutuamente y el espacio urbano-arquitectónico, su configuración y calidad juegan un rol muy importante en cómo nos conducimos y comportamos. Así un espacio urbano oscuro, solitario y abandonado resulta atractivo para la delincuencia y la violencia, sin embargo para muchas mujeres no hay otros caminos y tampoco alternativas. Es aquí donde es de suma importancia la intervención y el mejoramiento a través de un diseño pertinente, un equipamiento y habilitación de calidad y principalmente una gestión del espacio público adecuada con verdadero compromiso y voluntades desde distintos ámbitos que conlleven a soluciones integrales a la problemática aquí planteada.

\section{Bibliografía}

Bolos, S. (2008) mujeres y espacio público. Construcción y ejercicio de la ciudadanía. Universidad Iberoamericana, México DF.

Casados 2017 Violencia contra las mujeres en Veracruz . UVserva, No. 4. (2017).

Durán, M. A. (2000). Ciudades Proyectadas. En M. L. Penelas, La ciutat de les dames (págs. 63- 79). Barcelona, España: Centro de Cultura Contemporánea de Barcelona.

Durán, M. A. (2008 (primera edición 1998)). La ciudad compartida. Santiago de Chile: Sur Muxí, Z. 2015 Mujeres haciendo ciudadesaprendiendo del pasado: Kultur: revista interdisciplinària sobre la cultura de la ciutat, , Vol. 2, No. 3, 2015

Falú, A. (2009) Mujeres en la ciudad De violencias y derechos. Red Mujer y Hábitat de América Latina Ediciones SUR. Chile. 